\title{
Massenmedien im Dienst der Evangelisation: Kritische Bemerkungen zu einem römischen Dokument
} von Giselbert Deussen

Die nächste Bischofssynode in Rom, die im Oktober 1974 beginnen soll, wird sich mit dem Thema „Die Evangelisierung der heutigen Welt" beschäftigen. Die römischen Bischofssynoden sollen in der nachkonziliaren Kirche der Kommunikation $\mathbf{z w i s c h e n ~ d e r ~ r o ̈ m i s c h e n ~ Z e n t r a l e ~ u n d ~ d e n ~ v e r s c h i e d e n e n ~ T e i l k i r c h e n ~ d i e n e n . ~ W i r k u n g ~}$ und Eigenschaft dieser Kommunikation wären eine eigene Untersuchung unter speziell publizistischen Aspekten wert: Ist sie doch nicht Selbstzweck, sondern sie will Initialzündung neuen und Belebung des schon vorhandenen Informationsaustausches über Leben und Glauben der Kirche sein. Die kommende Synode wird sich dieser Problematik desto bewußter werden, als sie sich explizit mit den kirchlichen Kommunikationen nach innen und nach außen befassen will, wenn zwar auch „nur“ unter dem Aspekt der Evangelisation, ein Aspekt, der aber nach dem theologischen Selbstverständnis der Kirche ihr zentrales Anliegen oder - theologisch gesprochen ihre "Sendung" beschreibt.

\section{Das römische Dokument}

Rom wünscht $\mathrm{zu}$ diesem Thema keine exklusiven theoretischen Abhandlungen, sondern eine möglichst breite Basisdiskussion. Um diese Diskussion in Gang zu bringen, veröffentlichte das Sekretariat der Bischofssynode ein erstes Dokument, das neben rein akademischen Problemstellungen wohltuend viele praktische Fragen aufwirft. $\mathrm{Zu}$ diesen Sachfragen sollen nun die nationalen Bischofskonferenzen nicht von sich aus, sondern lediglich als letztinstanzliche "Redaktion “ des öffentlichen Basisgesprächs (in Seelsorge- und Priesterräten, in verschiedenen Kommissionen und Verbänden und den vielen Pfarrgremien) ihre Stellungnahmen bis Ende Februar 1974 dem Sekretariat der Bischofssynode in Rom zugestellt haben. Auf Grund dieser „Antworten" soll eine Synthese als Synodenunterlage erstellt werden.

Schon bei einer ersten Lektüre fällt angenehm auf: In diesem Dokument fehlt der apodiktische Ton, der theologischen „Papieren“ oft anhaftet. Es dominiert vielmehr die vorsichtige Frage, die natürlich aus bestimmten, ihrerseits zu befragenden Verständnishorizonten und Intentionen kommt. Hier soll nun der Fragen- und Vorschlagskomplex herausgegriffen werden, der die Evangelisation auf die Massenmedien und ihre vermeintlichen und tatsächlichen Funktionen bezieht.

Doch ein Inhaltsüberblick über das gesamte Dokument soll zunächst ein besseres Verständnis für die Zusammenhänge ermöglichen. Nach einer kurzen Einleitung, die eine theologische Definition des Begriffs "Evangelisation" unternimmt, gibt der 1. Teil einen Gesamtüberblick über die Evangelisation bei der heutigen Weltlage, der notwendigerweise sehr allgemein ist und willkürlich Momente referiert, die der

Dr. Giselbert Deussen ist regelmäßiger Mitarbeiter von CS. Nach dem Studium der Theologie, Sozialwissenschaft und Publizistik promovierte er mit einer Arbeit über die "Ethik der Massenkommunikation bei Papst Paul VI.“. Er ist Pressereferent der Konrad-AdenauerStiftung, Bonn. 
Evangelisation förderlich oder hinderlich sein können. Besonders die „Hindernisse in der Kirche selbst" lassen gewisse (konservative?) Fragesteller vermuten, die aber sympathischerweise hinter ihre Befürchtungen jeweils Fragezeichen setzen.

Der 2. Teil referiert sodann einige theologische Prinzipien vornehmlich des Vaticanum II. Beachtenswert sind hier die "Antinomien der Evangelisierung, die in eine Synthese zu bringen sind “, wobei mit einer in einem römischen Dokument verblüffen. den Aufgeschlossenheit wenigstens indirekt eine Pluralität der verschiedenen Theologien anerkannt wird.

Der praxisbezogene 3. Teil befaßt sich mit dem Wie der Evangelisation. Hier interessieren besonders die beiden Abschnitte, die das Verhältnis von Massenkommu* nikation und Evangelisation ansprechen. ${ }^{1}$ Unter Punkt E des mit „Einige Orientierungspunkte“ überschriebenen 1. Abschnitts heißt es:

"Da die öffentliche Meinung heute zum großen Teil durch die sozialen Kommunikationsmittel (Presse, Kino, Radio, Fernsehen usw.) geformt und geleitet wird, muß die Kirche in diesen Medien präsent sein. Die Nutzung der Kommunikationsmittel schließt ein:

1. die Prä-Evangelisation, d. h. eine genaue Kenntnisnahme der christlichen Glaubenslehre, der christlichen Ethik, des Verhältnisses zwischen Kirche und Welt u.s.w.;

2. Hilfe bei der Verkündigung des Evangeliums, damit die Katechese und Predigt durch die audiovisuellen Mittel eine Erläuterung erfahren und, der Kultur unserer Zeit entsprechend, durch Bilder in das Bewußtsein der Menschen eindringen;

3. die direkte Nutzung zur Verkündigung des Evangeliums, so daß die Verkündigung auch in jene Bereiche einzudringen vermag, die ihr für gewöhnlich verschlossen sind, und dies noch dazu so häufig, wie es der unmittelbaren Verkündigung nicht möglich ist."

Unter dem Punkt E des 2. Abschnitts „Einige praktische Vorschläge“ heißt es dann: „1. Sorgt man dafür, daß die Kirche (nach Möglichkeit) im Bereich der sozialen Kommunikation Einfluß hat?

2. Ist die Information über das Leben der Kirche genau und interessant? Werden Fehler in den allgemeinen Sendungen richtiggestellt?

3. Sorgt man für religiöse Sendungen (bzw. Spalten), die den verschiedenen Altersstufen und Verhältnissen der Menschen angepaßt sind?

4. Werden fähige Gläubige für die Arbeit in den Massenmedien ausgebildet?

5. Versucht man, im christlichen Volk einen kritischen Sinn gegenüber dem Angebot der Massenmedien auszubilden?"

\section{Evangelisation und „Einflu $\beta^{\star}$}

Das durchgängige Motiv, im Zusammenhang mit Evangelisation auch von den Kommunikationsmitteln zu sprechen, ist ihre Nützlichkeit. Die Schlußfolgerung, die daraus gezogen wird, heißt: Die Kirche muß in den Massenmedien Einfluß haben. Die Absichten der Kirche sind durchaus edel, aber keineswegs selbstlos. So unverhüllt und entwaffnend naiv spricht das Dokument von diesen Absichten, daß jeder mit 
den Massenmedien Befaßte - ob er der Kirche nun wohlgesonnen ist oder nicht weiß: Die Kirche will diese Mittel besitzen, um sie für ihre $Z$ wecke, die das Dokument mit Prä-Evangelisation, Verkündigen des Evangeliums, Predigt und Katechese beschreibt, einzusetzen. Angesichts dieser Absichtserklärung fragt sich nun gerade der Unvoreingenommene: Ist das alles? Mehr hat die Kirche unter ihrem Zentralanliegen Evangelisation im 20. Jahrhundert nicht zu den Massenmedien zu sagen? Erschöpfen sich ihre Fragen nur darin, wie man in den Besitz und die bessere Verwendung dieser Mittel kommt? Ist sie ein Aufpasser, der nur negativ dahinter her ist, wie seine Schäfchen durch diese Mittel keinen Schaden nehmen können? Will sie sich in die wahrhaftig schon allzu große Zahl jener einreihen, die machtlüstern in den Besitz jener Mittel kommen wollen, um ihre Zwecke zu verfolgen, Zwecke, die allüberall auf der Welt von Führern und Verführern unter dem Zauberwort "Einfluß" subsumiert werden?

Zudem: Warum schauen die Verfasser des Papiers immer noch - ist die Pastoralinstruktion "Communio et Progressio" bereits vergessen?" - nur auf jene längst nicht mehr so recht geglaubte Möglichkeit der Publizistik, die sie mit "Formen" und "Leiten" umschreiben?

\section{Marginalität als Hauptsache: Herrschaftsanspruch gegen Dienstleistung}

Wesen und Funktion der Massenmedien, ja der sozialen Kommunikationsmittel überhaupt, werden durch die Fragen des römischen Arbeitspapiers gar nicht angepeilt. Nur die marginalen und zweifelhaften Aspekte des Rechts auf Nutzung und Einfluß werden zu Themen der Beziehung von Evangelisation und Kommunikationsmitteln gemacht. Die Präsenz der evangelisierenden Kirche wird nur unter dem Aspekt des Nutzens motiviert und gibt sich somit als platter Utilitarismus, eine Haltung, die in der kirchlichen Morallehre zwar immer als häretisch verurteilt wurde, in der kirchlichen Praxis aber anderswo wie hier fröhliche Urständ feiert.

Die Abwesenheit von Fragen und Problemen, die etwaige Funktionen und Aufgaben der Massenmedien in Gesellschaften zum Ausdruck bringen, die sich in Entwicklung befinden und in denen die Kirche evangelisiert, ist erschreckend. Dies betrifft vor allem die sogenannten Missionsländer. Wenn sich moderne Missionsarbeit nach dem Verständnis der nachkonziliaren Kirche in dem Sinne als Kommunikation versteht, daß sie den zu missionierenden Völkern zu Entwicklung und Selbstverwirklichung in Christus verhelfen will, dann hat sie doch wohl zuallererst für ein gutes Funktionieren der in diesen Völkern neu entstehenden Kommunikationssysteme und -strukturen ihren Dienst anzubieten. Ich denke da z. B. an die für die Entwicklungsländern so wichtige interpersonale Kommunikation und ihre Einordnung in die ebendort in Aufbau befindlichen Massenkommunikationssysteme. ${ }^{3}$ Diese traditionelle Mensch-zu-Mensch-Kommunikation steht unversehens weltumspannenden Kommunikationsapparaturen gegenüber. Die Mission hätte hier zuerst einmal einen Beitrag zur Bewältigung der entstandenen Kommunikationskonflikte zu leisten, ehe sie an den eigenen Nutzen und Einfluß denken darf. Hier müßte vom "Dienen“ und nicht vom "Benutzen“ gesprochen werden. Ansätze für die Bereitschaft und die entsprechende Selbstlosigkeit, die dieser Dienst an der Kommunikationswirklichkeit von den Evangelisatoren verlangt, sind in dem römischen Dokument nicht zu finden. 


\section{Medienkolonialismus}

Kein Wort handelt von der Notwendigkeit, daß eine evangelisierende Kirche in den Ländern der Dritten Welt vor allem publizistische Entwicklungshilfe zu leisten hat. In diesem Zusammenhang ist die irreführende und verfälschte Verwendung des Begriffs der „Prä-Evangelisation“ anzunehmen, der als „genaue Kenntnisnahme der christlichen Glaubenslehre " etc. definiert wird. Genau dies aber würde ich als PostEvangelisation allenfalls aber als Evangelisation bezeichnen. Es wird also der zweite vor dem ersten Schritt getan, wenn unter Prä-Evangelisation jener Prozeß der Milieubildung und der Bodenbereitung verstanden wird, „der einer eigentlichen Evangelisation als Eingliederung in die christliche Gemeinschaft vorausgeht ". ${ }^{4}$

Gerade der Prä-Evangelisations-Bereich aber dürfte für den kirchlichen Dienst der publizistischen Entwidklungshilfe in Frage kommen. Doch das gebannte Starren auf den vermeintlichen Nutzen der Kommunikationsmittel verstellt den Blick für die Kommunikation der Gesellschaft und ihre Bedürfnisse überhaupt. Die daraus resultierende Haltung, wie sie sich in dem römischen Dokument exemplarisch als Abwesenheit des genannten wesentlichen Aspektes darstellt, könnte man mit „Medienkolonialismus" bezeichnen, der die Massenmedien egoistisch nach eigenen Zweckmäßigkeiten gebraucht und nicht nach denen der Gesellschaft. Der kirchliche Medienkolonialismus geht von einer abendländisch-snobistischen Post-EvangelisationsMentalität aus, verpaßte also die so wesentliche erste Stufe der Prä-Evangelisation, die der römischen Kirche sehr viel Selbstlosigkeit, Abstinenz von Eigeninteressen und ein waches Einfühlungsvermögen abverlangt. Eine Folge dieser Mentalität sie scheint auch in dem Papier auf - besteht dann darin, daß der Besitz von Maschinen und die Möglichkeit zu drucken, sowie der Besitz von Radio- und Fernsehstationen manchmal als Selbstzweck erscheinen. ${ }^{5}$ Am Rande sei erwähnt, daß diese Haltung die kirchliche Publizistik in den "christlichen " Industrieländern zu einer Getto-Publizistik hat verkümmern lassen. Warum ist man nun dabei, in der Missionsarbeit die gleichen Fehler zu wiederholen?

\section{Die Theologie als Hindernis}

Vor allem scheint für diese Art von Medienstrategie eine veraltete theologische Sicht von Evangelisation bzw. Mission, die auch in dem vorliegenden Dokument allgegenwärtig ist, verantwortlich zu sein. ${ }^{\circ}$ Diese geht zwar hurtig daran, mit neuen Begriffen eine neue Dimension moderner Weltbewältigung sich einzuverleiben. Aber sie muß in der Praxis notwendig scheitern, weil mit modernen Begriffen allein sich die Welt weder bewältigen noch einverleiben läßt, wenn sich die Haltung, die Mentalität, nicht ändert.

Denn es ist in der Tat sehr verdächtig, daß in einem kirchlichen Dokument, das Orientierungspunkte zum Thema Evangelisation setzen will, zu dem Problem Massenmedien nur die Begriffe Nutzen und Einfluß auftauchen. Rückschlüsse auf den theologischen Begriff der Evangelisation müßten von diesen beiden Begriffen einer eher undemokratischen Medienpolitik her fatal sein, da man eventuell die Annahme revidieren müßte, die Kirche habe jene verhängnisvolle Epoche ihrer Missions-

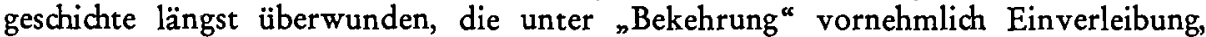
Unterwerfung und eine geistliche Variante des politischen Begriffs Kolonialismus verstand. Es bleibt zu hoffen, daß die Bischofssynode mehr und anderes zu dem Verhältnis von Evangelisation, Kommunikation und Massenmedien zu sagen hat als dieses vom Sekretariat veröffentlichte erste Dokument. 


\section{Anmerkungen:}

1. Ich zitiere nach einer Ubersetzung des römischen Dokuments in amtlicher, vom Sekretariat zur Verfügung gestellter Ubersetzung, veröffentlicht in: „Schweizerische Kirchenzeitung“ 141. Jg., 38/1973 vom 20. September, S. 573-579.

2. Vgl. z. B. "Communio et Progressio" Nr. 16, in: Nachkonziliare Dokumentation Bd. 11, Paulinus-Verlag Trier, 1971, S. 165. Hier wird dazu aufgerufen, das Gesamtangebot der verschiedenen Kommunikationsmittel in dem Sinne zu gebrauchen und nach den Wirkungen zu beurteilen, „inwieweit es als solches dem Gemeinwohl dient, d. h. wie es durch Information, Bildung und Unterhaltung das Leben und die Entwidklung der betreffenden Gesellschaft fördert" .

3. Vgl. Franz-Josef Eilers: Die Pastoralinstruktion zur Kommunikation in den Missions- und Entwicklungsländern, in: ders. u. a. (Hrsg.): Kirche und Publizistik. Dreizehn Kommentare zur Pastoralinstruktion "Communio et Progressio“, München-Paderborn-Wien 1972, S. $82 \mathrm{f}$.

4. Franz-Josef Eilers: Zur Bedeutung der Publizistik in der Missionsarbeit heute, in: $N$ Neue Zeitschrift für Missionswissenschaft ${ }^{\text {c }}$ 28:1972, Heft 4, S. 246.

5. Vgl. ders.: a.a.O., S. $250 \mathrm{f}$.

6. Der Begriff der Evangelisation in diesem Dokument müßte deshalb unter theologischen Aspekten einmal sehr gründlich befragt und neu gefaßt werden.

\section{S U M M A R Y}

Having studied strategy of communication, especially of Pope John XXIII and Paul VI, the author states a series of critical remarks on the first document of the Secretariate of the Bishop's Synod, 1974 on the theme, "Evangelisation of Today's World". He pidks up questions and proposals on evangelisation with regards to mass media, and their actual and pretended functions. The reproach to this complex is, it puts the "influence" above the "forming" and "guiding" as well as "dominating" before "service" thus falling back into a kind of "mass media colonialism".

\section{RESUM É}

Faisant usage de sa connaissance, justement aussi en matière de stratégie de communication des Papes Jean XXIII et Paul VI dans le domaine spirituel, l'auteur se permet ici des remarques critiques au sujet du premier document du secrétariat du synode 1974 des évêques ¿ Rome ayant pour thême: "L'Evangélisation, aujourd'hui, dans le monde“. Il choisit au hasard dans le complexe de questions et de suppositions qui met en rapport l'Evangélisation et les moyens de communication, leurs fonctions présumées et effectives. Il reproche à ce complexe de mettre l', influence“ sur la "forme“ et sur la "conduite“, de placer le „droit de domination" avant la "prestation de service“ et de retomber dans une sorte de "colonialisme des moyens".

\section{RESUMEN}

Basándose en sus conocimientos y precisamente en la estrategia para los medios de comunicación eclesial de los papas Juan XXIII y Pablo VI, el autor se permite anotaciones críticas al primer documento del Secretariado del Sínodo romana de los obispos 1974 sobre el tema -La evangelización del mundo actual“. Analiza el complejo de problemas y propuestas que la evangelicación plantea a los medios de comunicación y a sus funciones hipotéticas y efectivas. Recrimina a este complejo el imponer la "influencia" sobre el "conformar" y el "dirigir", el anteponer las "pretensiones de soberanía“ a la „prestación de servicios" y de retroceder a una especie de "colonialismo de los medios de comunicación ${ }^{\circ}$. 